\title{
Mobile Phone Support for Rural Health Workers in Nepal through 'Celemedicine'
}

\author{
Joanna Morrison, ${ }^{1}$ Naba Raj Shrestha, ${ }^{1}$ Bruce Hayes, ${ }^{1}$ Mark Zimmerman ${ }^{1}$ \\ 'Nick Simons Institute, Sanepa, Lalitpur, Nepal.
}

\begin{abstract}
Globally, there is a shortage of health workers in rural areas. Effective health systems depend on having sufficient, accessible health workers with the right skills. In countries like Nepal, highly skilled health workers often prefer to work overseas or in urban centres, and therefore, in the short term, it may be pragmatic to focus on ensuring support and skills development of mid level or paramedical health workers. Information technology has the potential to support these health workers. We describe a pilot intervention undertaken in Gulmi District, whereby all mid level health workers in the district have been provided with a free phone number to call three General Practitioner Doctors (GPs) in the District Hospital. The intervention aims to increase appropriate referral, and increase connectivity between the District centre and peripheral health facilities. We hope that our intervention will provide support to rural health workers, and, if implemented as part of a package of interventions, may increase retention. We present some initial findings from discussions with health workers and analysis of call-log data, and describe our next phase evaluation and possible scale-up.
\end{abstract}

Keywords: human resources; mobile phone; mhealth; Nepal; retention; rural.

\section{INTRODUCTION}

Where there are more health workers, there are better health outcomes. ${ }^{1}$ In many low-income countries, there is a glut of health workers in urban areas, leaving rural areas underserved. Nepal is no different: 83 per cent of the population lives in rural areas, ${ }^{2}$ yet health care is accessed more in urban areas. For example, only 32 per cent of births in rural areas were attended by a doctor, nurse or midwife, compared to 73 per cent of deliveries in urban areas. ${ }^{3}$ A study conducted mainly in the west of Nepal in 2006 found that out of the 23 district hospitals surveyed, 39 out of 50 doctors were present at the time of data collection. Of those, only 22 (56 per cent) had been present for 12 months of the year and absenteeism rates were high. Auxiliary Health Workers and Auxiliary Nurse Midwives (paramedical health workers) are more likely to be in post in rural areas than higher-level cadres. ${ }^{4}$ In the short term, therefore, it may be pragmatic to focus on ensuring support and skills development of mid level or paramedical health workers ${ }^{5}$ It is important to find ways of increasing the efficiency of existing health workers while maintaining high quality health service provision and job satisfaction. ${ }^{6}$ Studies have shown that in Nepal, job satisfaction

Correspondence: Dr. Joanna Morrison, jmorrison@nsi.edu.np Nick Simons Institute, Box 8975; EPC 1813, Sanepa, Lalitpur, Nepal. 
depends partly on rural health workers' access to professional and skill development, ${ }^{7,8}$ their working conditions, and functionality of health management systems. Information technology may have a role to play in addressing some of these issues..$^{9,10}$

Telemedicine and mobile health

Telemedicine is the delivery of health care and sharing of medical knowledge using telecommunications, and may include images as well as voice and message communication. There has been a lot of optimism about the potential for telemedicine interventions to reach marginalised groups in low-income countries. For health workers, this type of intervention can facilitate cooperation between health workers from better-served areas and those in underserved areas dealing with issues of professional isolation, providing an opportunity for continuing medical education. ${ }^{11,12}$

\section{Telemedicine in Nepal}

Telemedicine has been piloted in Nepal, to support doctors in rural hospitals ${ }^{13} \mathrm{~A}$ WHO report describes a recent venture in three districts of Nepal, whereby rural doctors were advised by specialists in Kathmandu. ${ }^{14}$ This pilot project with doctors ran from 2006 to 2009 in three district hospitals. Some positive results were reported but implementers were concerned about the lack of technical capacity of participants and their managers, and the rural doctors' fear of losing professional autonomy. ${ }^{15}$ Other pilot ventures have had some success, ${ }^{16-18}$ and may provide useful lessons for national integration of telemedicine within the public health system. The Government of India, through the SAARC telemedicine network has supported a national telemedicine network in Nepal, through Patan Hospital in Kathmandu. ${ }^{19}$ In 2011 it was reported that 25 hospitals had a telemedicine facility, ${ }^{20}$ with a reported expansion to five more districts in 2012.21 Use of visual technology has been limited, and the success of the email response system for specialist cases has not been formally evaluated. The government has also been dealing with problems of internet capacity in district hospitals, and the availability of electricity. The sustainability of telemedicine to enhance rural health care depends partly on full integration of systems into service provision, and therefore does not only rely on the interest and commitment of a few individuals. Future evaluations should identify the optimum and minimum conditions for successful telemedicine interventions, measure their impact on health outcomes, and their potential for sustainability in the Nepalese context.

\section{Mobile phones for health (mhealth)}

$90 \%$ of the world's population are covered by a mobile phone signal, and there are 5.3 billion subscribers ${ }^{22}$, and while telecommunications infrastructure is often too slow and too expensive to connect computers to the internet, low bandwidth applications for mobile phones enable connection through mobile networks. ${ }^{23}$ The application of mobile telephones for medical and public health - mHealth ${ }^{24}$ - has been used for surveillance and monitoring, ${ }^{25,26}$ mass communication, ${ }^{27}$ and increasing access to information. ${ }^{28}$ Through the use of mobile phones and Personal Data Assistants, health workers can collect information on patients that can be digitally stored and shared, enabling appropriate and timely responses to disease epidemics, or to individuals in need of additional treatment. Systematic reviews of studies mostly conducted in high-income countries have shown that Short Message Service (SMS) reminders to patients increases their attendance at health care appointments. ${ }^{29,30}$ Although much of the literature has focussed on high income countries, researchers in the field of HIV/AIDs have been piloting and evaluating Short Message Service (SMS) technology in low income countries to improve patient adherence to treatment programmes. ${ }^{23,31,32}$ For general health promotion, SMS messaging has also been used to raise awareness through one-way communication of health messages and through more innovate approaches using interactive quiz methods. ${ }^{23}$ The mHealth Alliance has been set up to facilitate the sharing of knowledge about pilot projects and their effectiveness in low income countries, yet there have been few evaluations of interventions implemented at scale. ${ }^{33}$ For example in Uganda, in 2008 and 2009 approximately 23 out of 36 mhealth initiatives did not move beyond the pilot phase. ${ }^{34}$ Little is known about the effectiveness of mhealth interventions. Scale-up of mhealth interventions should be preceded by efficacy and effectiveness trials so that they are founded on an appropriate evidence base. Randomised controlled trials of mhealth interventions that can evaluate the multiple features of interventions have also been recommended. ${ }^{35}$

\section{Mobile phone support to health workers}

Mobile phone interventions have also been used to support health workers diagnosing and treating patients. A study by Mahmud et al. at a rural hospital in Malawi evaluated an intervention where community health workers (volunteers) were given mobile phones to send text messages to their referral centres about any issue concerning their clients. ${ }^{36}$ They found that this intervention was cost effective, saving health worker time, and they were able to double their capacity of the Tuberculosis treatment programme. Chang's 
study in rural Uganda provided mobile phones to Peer Health Workers (PHWs) who were each monitoring 15-20 HIV positive clients. PHWs received a two day training on basic HIV pathogenesis, prevention, treatment, adherence counselling, performing pill counts, protecting patient confidentiality, and filling out a home visit form. They provided clinical and adherence monitoring and psychosocial support to patents at clinic and home visits. They were able to call within certain hours, and a medical officer with training in HIV care answered their calls. ${ }^{37}$ They tested the impact of the mobile intervention through a cluster randomised controlled trial, and found that the intervention had no effect on primary outcomes of cumulative risk of virologic failure at 24 weeks nor secondary outcomes of, patient adherence, virologic failure at 24-48 weeks, loss to follow-up or mortality. Although there were problems of collecting data on patient adherence, and the study was not powered adequately, there was little indication that the intervention would have impacted on the outcomes chosen. Qualitative data collected in this study indicates that the Peer Health Workers and health workers felt the intervention had a positive impact on patient care, access to medical advice, easy re-supply of drugs, confidence of PHWs, and improved relations between PHWs and health workers. Text messaging was perceived to be slightly less effective than phone conversations, although some PHWs felt that this may have increased adherence with patients becoming more aware of reporting systems. ${ }^{38}$

\section{A mobile phone intervention for mid level health workers in Nepal}

Telemedicine is sometimes not an option in remote primary health care facilities with no computers or internet access. Yet mobile phone use in Nepal is high. Nine out of ten households in urban areas, and seven out of ten households in rural areas have a mobile phone. ${ }^{36}$ After considering the experience with telemedicine in Nepal, we have developed a simple intervention that uses mobile phones to support paramedical staff in in Sub-Health Posts, Health Posts and Primary Health Centres of rural Nepal. Our experience suggests that interaction between health workers in rural areas and the district headquarters is minimal and our intervention aims to increase professional linkages between doctors in the district hospital and the periphery.

\section{METHODOLOGY}

Our mobile phone intervention - celemedicine - connects mid level health workers to General Practitioner Doctors (GPs) in their district hospital through a free phone facility. We phoned all participating 24 health workers and three GPs to inform them about the intervention. In our pilot, these mid level health workers can use any phone - not necessarily a mobile phone - to contact any one of three GPs in the district hospital. They dial a free phone number, and when connected, dial a three-digit number to connect to a GP. Each GP has a different number, so that identifying GPs for followup calls is easier. Phone calls are routed through our office in Kathmandu for monitoring purposes, and GPs receive the call on their mobile phone. Two of the GPs participating in this pilot are bonded to work in a rural hospital for three years after NSI gave them scholarships to specialise as a GP. For piloting purposes, we are implementing this intervention in Gulmi district, which is in the hills where mobile phone coverage is good. Our pilot sought to explore the technical feasibility of our intervention, and its acceptability among patients, mid level health workers, and GPs, with a view to scalingup this intervention to more health workers, and other districts.

\section{Piloting results}

We reviewed call data from $6^{\text {th }}$ September to $31^{\text {st }}$ October 2011, and found that 16 out of 24 health workers had called a GP. A total of 71 calls were made. Fifty-nine per cent (42) of calls were made between $9 \mathrm{am}$ and $5 \mathrm{pm}$, and one doctor usually received out-ofhours calls (Table 1). We conducted a telephone survey with those health workers that had called the GP.

Overall, mid level health workers and patients were positive about the intervention. They reported that they felt more supported, and the consultation helped them to make decisions in serious cases. Usually the phone call to the GP resulted in referral. The patient was usually referred to the Mission Hospital in a neighbouring district, as opposed to Gulmi District Hospital. This may be because the mission hospital has integrated care facilities and may be closer in some cases than the district hospital in Gulmi. Also, we recognise that it takes time to increase confidence in district hospitals that were previously understaffed.

Health workers usually consulted GPs while they were with patients, and sometimes the patient listened to the GP through the speakerphone. Sometimes, when health workers were unable to connect through the free phone line, they called the GP directly. Although this is a positive development, this makes the impact of our intervention more difficult to monitor, and we need to capture the extent to which health workers would have done this without the intervention. Four respondents felt that specialist doctor consultation would be helpful. Very few respondents reported technical difficulties in making calls, but this could be due to the fact that 
they are used to receiving interrupted service, caused by network overload, and frequent power cuts. Setting up and maintaining the system has been easy, but our reliance on the national telecommunications network makes our system as vulnerable to network problems as any other phone communications.

\section{DISCUSSION}

\section{Scale-up and evaluation}

On the basis of our successful pilot we have scaled up our intervention, which now includes 147 mid level health workers in Gulmi, now including Auxiliary Nurse Midwives. There is a focal person in the district headquarters who is responsible for informing health workers and encouraging use of the free phone facility. We have added phone lines to deal with the anticipated increase in calls, and we will conduct an evaluation after 6 months of implementation in order to explore the relevance, acceptability, affordability and effectiveness of the intervention. ${ }^{6}$ We will seek ethical approval, and our study will involve users, non-users and GPs in evaluating celemedicine. We will describe how and when celemedicine is used, and ask stakeholders to describe their experience with celemedicine, and how it is affecting their work and patient flow. We will also describe the cost of this intervention, presenting the total costs, and the cost of scaling-up this intervention at a national level. We will also model the proportion of the health budget that this would constitute. On the basis of this research we have planned to adapt the intervention and pilot in a more remote district (Kalikot), and will explore the possibility of conducting a 'before and after study', looking at the impact of celemedicine on utilisation and referral.

The success of our intervention depends on the motivation of mid level health workers to use the service, the willingness of the doctors to offer advice, and the willingness and ability of patients to take that advice. Community barriers to accessing care are well

for retention of rural health workers. documented in the field of maternal health ${ }^{(39)}$, and celemedicine does not deal with financial and other constraints to acting on referral advice. Therefore we should be cautious about the potential impact this intervention may have on utilisation of services at District Hospitals.

The workload of the GP will increase as celemedicine is utilised more, and it will be interesting to see how GPs in Gulmi deal with this. An allocated time to receive calls from the periphery may be of benefit if the number of calls substantially increases. Yet this has the disadvantage of limiting the usefulness of celemedicine in supporting peripheral health workers in emergencies. Confidentiality of data is cited as a concern in many of the studies, particularly those with HIV/AIDs interventions, and in the UK, there are worries over a potential breakdown in communication between doctors and patients. This may not be a concern in Nepal, and we hope that this intervention will enable more communication between doctors and patients in rural areas.

\section{CONCLUSION}

There is potential to use technology to address human resources for health issues in low-income countries. Innovative efforts such as this celemedicine intervention need to be rigorously evaluated to explore their ability to improve retention of rural health as part of a broader package of support. We hope that our approach using mobile phone technology will support linkages between health workers, reduce professional isolation of peripheral health workers, and increase appropriate referral. We will share our knowledge and experience of this intervention post evaluation, and we hope to encourage innovation to deal with human resources for health issues in rural Nepal. Through our work with the Government of Nepal in District Hospitals such as Gulmi, we hope to foster local ownership of the evidence about what works, and what doesn't work,

\section{REFERENCES}

1. WHO. Increasing access to health workers in remote rural areas through improved retention: Background Paper. Geneva: World Health Organisation; 2009.

2. National Planning Commission. Population Census Preliminary Report. Kathmandu: Central Statistics Department, National Planning Commission, Government of Nepal; 2011.

3. Nepal Ministry of Health and Population. Nepal Demographic and Health Survey: Preliminary Report. Kathmandu: Government of Nepal, New Era, ICF Macro, USAID; 2011.

4. Nick Simons Institute. Deployment of health workers in government district hospitals in Nepal. Kathmandu, Nepal: Nick Simons Institute; 2006.

5. WHO. Task Shifting: rational redistribution of tasks among health workforce teams: global recommendations and guidelines: Geneva; 2008.

6. WHO. Increasing access to health workers in remote and rural areas through improved retention: Global policy recommendations. Geneva: WHO; 2010.

7. Butterworth K, Hayes B, Neupane B. Retention of general practitioners in rural Nepal - a qualitative study. Australian journal of rural health. 2008;16:201-6. 
8. Kolehmainen-Aitken $\mathrm{R}$, Shrestha I. Human Resources Strategy Options for Safe Delivery. Kathmandu: Ministry of Health and Population, Government of Nepal, Support for Safe Motherhood Programme, RTI International; 2009.

9. UNDP. Regional Human Development Report: Promoting ICT for Human Development in Asia: Realizing the Millennium Development Goals. New Delhi: Elsevier; 2005.

10. WHO. Information technology in support of health care. Department of essential health technologies; downloaded 12.12.11.

11. Gagnon M, Duplantie J, Fortin J, Landry L. Exploring the effects of telehealth on medical human resources supply: a qualitative case study in remote regions. BMC Health Services Research. 2007;7(6).

12. WHO. Strategy 2004-2007 eHealth for Health-care deliveryundated.

13. Graham L, Zimmerman M, Vassallo D, Patterson V, Swinfen $\mathrm{P}$, Swinfen R, et al. Telemedicine - the way ahead for medicine in the developing world. Tropical Doctors. 2003;33(1):36-8.

14. Roodenbeke Ed, Lucas S, Rouzaut A, Bana F. Outreach services as a strategy to increase access to health workers in remote and rural areas. Geneva: WHO; 2011.

15. Pradhan M. Telemedicine in Nepal. In: Wootton R, Patil N, Scott R, Kendall H, editors. Telehealth in the developing world. London: Royal Society of Medicine Press Ltd; 2009. p. 182-92.

16. Piya R. Scope and challenges of telemedicine in Nepal: a look towards the future. Tromso: University of Tromso, Norway; 2010.

17. Nepal Wireless Networking Project. Nepal Wireless. Kathmandu: Nepal Wireless; 2012 [cited 2012 11th November]; Available from: http://www.nepalwireless. net $/$ index.php? option $=$ com_content\&view $=$ article\&id =116:about-us\&catid=34:info.

18. 18. Jha A, Gurung D. Reaching the unreached: a model for sustainable community development through information and communication technology. Journal of Nepal Medical Association. 2011;51(184):213-4.

19. 19. IRIB World Service. SAARC telemedicine centre to link Nepal with Indian cities. IRIB World Service. January 22nd 2011.

20. The Kathmandu Post. Balm to rural folk: 25 districts get telemedicine services. The Kathmandu Post. 22nd January 2011

21. The Kathmandu Post. Telemedicine services in 5 more districts. The Kathmandu Post. 21st August 2012.

22. Howitt P, Darzi A, Yang G-Z, Ashrafian H, Atun R, Barlow J, et al. Technologies for health. Lancet. 2012;380:507-35.

23. Leach-Lemens C. Using mobile phones in HIV care and prevention. HIV and AIDs Treatment in Practice. 2009(137):2-7.

24. van Heerden A, Tomlinson M, Swartz L. Point of care in your pocket: a research agenda for the field of $\mathrm{m}$-health. Bulletin of the World Health Organisation. 2012;90:393-4.
25. Morris K. Mobile phones connecting efforts to tackle infectious disease. Lancet. 2009;9:274

26. Freifeld C, Chunara R, Mekaru S, Chan E, Kass-Hout T, Ayala Lacucci A, et al. Participatory epidemiology: use of mobile phones for community-based health reporting. PLOS Medicine. 2010;7(12):e1000376.

27. Feder L. Report from the field: Cell phone medicine brings care to patients in developing nations. Health Affairs. 2010;29(2):259-63.

28. Kahn J, Yang J, Kahn J. Mobile health needs and opportunities in developing countries. Health Affairs. 2010;29(2):254-61.

29. Car J, Gurol-Urganci I, de Jongh T, Vokopivec-Jamsek V, Atun R. Mobile phone messaging reminders for attendance at health care appointments. Cochrane Database of Systematic Reviews. 2012;7:CD007458.

30. Guy R, Hocking J, Wand H, Stott S, H A. How effective are short message service reminders at increasing clinic attendance? A meta-analysis and systematic review. Health Service Research. 2012;47:614-32.

31. Lester R, Karanja S. Mobile phones: exceptional tools for HIV/AIDS, health and crisis management. Lancet. 2008;8:738-9.

32. Lester R, Ritvo E, Mills E, Kariri A, Karanja S, Chung M, et al. Effects of a mobile phone short message service on anti retroviral treatment adherence in Kenya (WelTel Kenya 1): a randomised trial. Lancet. 2010;376:1838-45.

33. Kaplan W. Can the ubiquitous power of mobile phones be used to improve health outcomes in developing countries? Globalization and Health. 2006;2(9).

34. 34. Lemaire J. Scaling up mobile health: elements necessary for the successful scale up of mhealth in developing countries. Geneva: Advanced development for Africa; 2011.

35. Tomlinson M, Rotheram-Borus M, Swartz L, Tsai A. Scaling up mHealth: Where is the evidence? PLOS Medicine. 2013;10(2):e1001382.

36. Mahmud N, Rodriguez J, Nesbit J. A text message-based intervention to bridge the healthcare communication gap in the rural developing world. Technology and Health Care. 2010;18:137-44.

37. Chang L, Kagaayi J, Nakigozi G, Galiwango R, Mulamba J, Ludigo J, et al. Telecommunications and health care: An HIV/AIDS warmline for communication and consultation in Rakai, Uganda. J Int Assoc Physicians AIDS care. 2008;7(3):130-2.

38. Chang L, Kagaayi J, Arem H, Nakigozi G, Ssempijja V, Quinn T, et al. Impact of a mHealth intervention for peer health workers on AIDS Care in Rural Uganda: a mixed methods evaluation of a cluster-randomised trial. AiDS and Behaviour. 2011;15(8):1776-84.

39. Pradhan A, Suvedi BK, Sharma SK, Puri M, Poudel P, Chitrakar SR, et al. Nepal Maternal Mortality and Morbidity Study 2008/2009. Kathmandu: Family Health Division, Department of Health Services, Ministry of Health and Population, Government of Nepal; 2010. 